\title{
The Influence of Hippocampal Lesions on the Discrimination of Structure and on Spatial Memory in Pigeons (Columba livia)
}

\author{
John M. Pearce, David N. George, Mark Haselgrove, Jonathen T. Erichsen, and Mark A. Good \\ Cardiff University
}

\begin{abstract}
Pigeons (Columba livia) were trained with a spatial structural discrimination, which was based on the spatial relationship among the components of a pattern, and a feature-binding structural discrimination, which was based on how different visual features within a pattern were combined. Neither discrimination was impaired by damage to the hippocampus and area parahippocampalis. The lesions impaired performance on a spatial working memory and a spatial reference memory task in open field. The results indicate an intact hippocampus is not essential for the solution of structural discriminations in pigeons and the hippocampus is important for processing some types of spatial information-that used in navigation, but not other types-that used in spatial structural discriminations.
\end{abstract}

Keywords: hippocampus, pigeons, discrimination, space, structure

Most discriminations between patterns of stimulation can be solved on the basis of the features they contain. The squares in the top left-hand panel of Figure 1 represent two patterns that might be used for a simple white-gray discrimination, and the lower two squares might be used for a horizontal-vertical discrimination. To master these discriminations, it is necessary to associate a basic feature of a pattern, such as its color, with the appropriate outcome. Even if the problem is made more complex by combining features to create the four patterns in the central panel of Figure 1, with food signaled by the patterns in the upper but not the lower row, the solution to the discrimination can still be based on the basic features of the patterns. For this biconditional discrimination, pairs of features indicate the outcome of a trial, with food being delivered when a pattern contains the color gray and vertical lines or the color white and horizontal lines, but not when it contains other combinations of these features.

The right-hand panel of Figure 1 shows two patterns that might be used for a more complex discrimination, in which the information provided by the presence or absence of basic features is insufficient for its solution. For this structural discrimination, subjects must discriminate between two patterns containing the same basic features but that are assembled in different ways. Each pattern contains two squares, one filled with horizontal lines and the other with vertical lines, and one containing the color white and the other the color gray. On the basis of the elements they contain, therefore, the patterns are indistinguishable, and in order to tell

John M. Pearce, David N. George, Mark Haselgrove, and Mark A. Good, School of Psychology, and Jonathen T. Erichsen, School of Optometry and Vision Sciences, Cardiff University, Cardiff, United Kingdom.

This research was supported by grants from the Biotechnology and Biological Sciences Research Council and the Medical Research Council of the United Kingdom. We are grateful to Tim Pearce for his assistance with the experiments and to Michael Colombo for his many helpful suggestions throughout the course of these studies.

Correspondence concerning this article should be addressed to John M. Pearce, School of Psychology, Cardiff University, Cardiff CF10 3YG, United Kingdom. E-mail: pearcejm@cardiff.ac.uk them apart it is necessary to bind together their basic features to create what might be called higher order features. Hence, it is the combination of the higher order features of gray-vertical lines and white-horizontal lines that signals food and the combination of gray-horizontal lines and white-vertical lines that signals the absence of food. George and Pearce (2003) demonstrated that pigeons can solve this type of discrimination, which, they argued, shows this species is capable of feature binding.

The patterns for a different type of structural discrimination studied by George, Ward-Robinson, and Pearce (2001) consisted of two adjacent circles separated by a small gap. The circles were of different colors, so that food might be presented on trials when a red circle was to the left of a yellow circle, but not when a yellow circle was to the left of a red circle. These patterns are composed of the same basic features, but they again differ in the manner in which the features are assembled. More specifically, it is the spatial relationship between the two colors that is important, and for this reason the task will be referred to as a spatial structural discrimination. Pigeons were able to solve this discrimination with little difficulty. For reasons that we make evident shortly, it was necessary for the design of both of these structural discriminations to be more complex than has just been implied.

If animals are able to solve structural discriminations, the question is then raised as to which region of the brain is responsible for this ability. In fact, there is relatively little evidence to draw upon in answer to this question. A few studies involving humans suggest the hippocampus might be involved in processing structural information. There is, for example, evidence that this region is involved in binding features that occur together. Kroll, Knight, Metcalfe, Wolf, and Tulving (1996) presented humans with lists containing words such as valley and barter and then asked them whether one of the words had been barley. Participants with damage to the left hippocampus were more likely than controls to respond in the affirmative. The implication of this result is that the participants with damage to the hippocampus were able to remember that they had been exposed to the syllables bar and ley, but they were unable to remember the syllables with which they had been paired during their original exposure to the list. It was therefore suggested that 


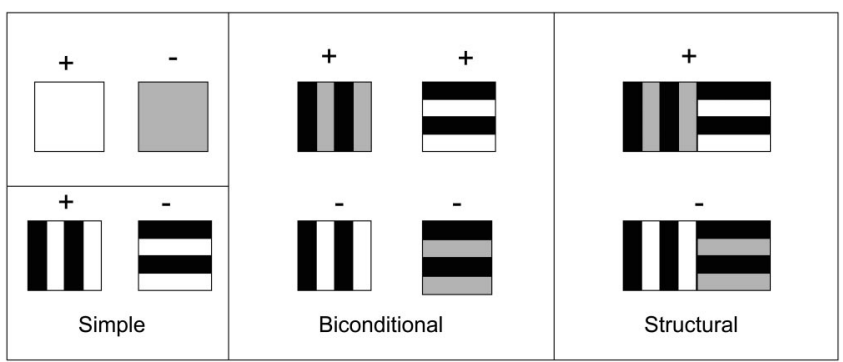

Figure 1. The different types of patterns that might be used for a simple, a biconditional, and a structural discrimination between reinforced $(+)$ and nonreinforced (-) patterns.

the hippocampus is important for binding the components of words together. Additional support for this conclusion can be found in studies of individuals with amnesia who have damage to the hippocampus (Mayes, Van Eijk, Gooding, Isaac, \& Holdstock, 1999; Vargha-Khadem et al., 1997). Such individuals are prone to treat novel objects constructed from arrangements of components from familiar objects as themselves being familiar, which implies that their memory of which features originally occurred together was poor (see Aggleton \& Pearce, 2002).

There have been rather few investigations of the ability of animals to solve structural discriminations, and it may not be surprising therefore that rather little has been said about the neural structures that subserve this ability in nonhumans. A number of authors have proposed that the hippocampus is important for the acquisition of configural or conjunctive representations (e.g., Fanselow, 2000; O'Reilly \& Rudy, 2001; Sutherland \& Rudy, 1989). In general, these proposals are concerned with how animals are able to discriminate between patterns composed of different features and not about how they might discriminate between patterns composed of the same features that are structured in different ways. Aggleton and Pearce (2002), on the other hand, proposed specifically that the hippocampus is important for the representation of structural information in animals (see also Eichenbaum \& Bunsey, 1995). One source of evidence that is consistent with this proposal comes from studies in which animals are repeatedly exposed to an array of three objects whose positions relative to each other, and to an external frame of reference, remain constant. They were then tested with a new configuration of the same objects. Such a test was found by Wan, Aggleton, and Brown (1999) to result in enhanced levels of Fos in the hippocampus and by Save, Poucet, Foreman, and Buhot (1992) to result in enhanced levels of exploratory activity directed toward the objects in normal subjects, but not those with damage to the dorsal hippocampus (see also Lee, Hunsaker, \& Kesner, 2005). Because the test trial involved rearranging the same objects that were present during training, one implication of these results is that the hippocampus is responsible for acquiring information about the spatial relationships between the elements of a configuration of objects-in other words, spatial structural information. On the other hand, in both studies the test trial involved presenting a familiar object in a new location. It is conceivable, therefore, that the hippocampus has rather little to do with acquiring knowledge about the structure of a configuration of objects and more to do with where they are located relative to some frame of reference. The proposal that the hippocampus serves as a cognitive map (O'Keefe \& Nadel, 1978) seems to be consistent with this second explanation for the findings of Wan etal. and Save et al.

The purpose of the present article is to examine the role of the avian hippocampus in the solution of structural discriminations. Pigeons have thus far proved in our laboratory to be more adept than rats at solving structural discriminations. Accordingly, pigeons were used for the two reported experiments. Not only has this species been shown to master, with relative ease, the two structural discriminations that have been described, but the available evidence suggests that the avian and mammalian hippocampus share a common functional attribute in terms of processing spatial information (Bingman, Jones, Strasser, Gagliardo, \& Ioalè, 1995; Colombo \& Broadbent, 2000; MacPhail, 2002).

\section{Experiment 1}

A group of pigeons with lesions of the avian homologue of the mammalian hippocampus, the hippocampus and the area parahippocampalis (Hp-APH), and a sham operated control group, were trained on a series of discriminations of increasing complexity culminating in two structural discriminations. If the hippocampus is involved in the perception of structure, then it was conceivable that both groups would perform similarly on the initial discriminations, but the group with damage to this region of the brain would be impaired when tested with the structural discriminations.

After being trained with a single stimulus, the two groups received simple discriminations to confirm that the lesions did not impair the ability to discriminate between two colors and between lines of different orientations. The stimuli were presented in a single square on a TV screen behind a response key, and food was presented after some patterns but not others. Colombo, Broadbent, Taylor, and Frost (2001) showed that hippocampal lesions have no effect on the acquisition of such simple visual discriminations. The two groups then received a biconditional feature discrimination similar to the one depicted in the central panel of Figure 1. Food was presented if the square contained vertical lines of Color A (AV) or horizontal lines of Color $\mathrm{B}(\mathrm{BH})$ but not if it contained the two other possible combinations of orientation and color (AH or $\mathrm{BV}$ ). To the best of our knowledge, this test constitutes the first attempt to assess the impact of Hp-APH lesions on the acquisition by pigeons of a biconditional discrimination. Given that a negative patterning discrimination is unaffected in this species by such lesions (Broadbent, Gallagher, \& Colombo, 1999), it is likely that the biconditional discrimination will also be unaffected. Next, the groups received a structural discrimination based on the patterns shown in the right-hand panel of Figure 1. One pattern was composed of vertical lines of Color $\mathrm{A}$ in the left-hand square and horizontal lines of Color B in the other square, AV-BH, whereas these features were rearranged for the other pattern, BV-AH. A simple way to solve this discrimination, without relying on structural information, would be to concentrate on one of the squaressay, the one on the left-and to use its color as the cue for determining the trial outcome. To forestall this strategy, the mirror images of the two patterns were used, BH-AV and AH-BV, with the mirror image signaling the same outcome as its parent. Even now it would be possible to solve the discrimination without referring to structural information. The problem could be solved by looking for lines of a particular orientation and then using their color to indicate whether or not food would be presented. To counter the development of this strategy, a third color, C, was 
introduced in order to create the 12 patterns that are listed in the upper panel of Table 1. In this table, by way of example, AV-BH refers to a pattern based on the design shown in the top of the right-hand panel of Figure 1, with a square containing vertical lines of Color A to the left of a square containing horizontal lines of Color B. To discriminate between any pattern in one column of the upper panel of Table 1, and its partner in the adjacent column, it would be insufficient to focus on a particular color or line orientation. Instead, the solution of the discrimination depends on subjects' binding together information about the color and orientations of the lines in each square. If the group with $\mathrm{Hp}-\mathrm{APH}$ lesions should solve this discrimination, then it would indicate that an intact hippocampus is not a necessary requisite for feature binding.

The effect of damage to the Hp-APH on the ability to solve a spatial structural discrimination was assessed in the next stage of the experiment. Both groups received a discrimination involving three colors, A, B, and C, and two adjacent circles. Thus, a circle filled with Color A to the left of a circle filled with Color B might signal food, and the mirror image of this pattern would signal the absence of food. Once again, if this discrimination is solved, it would not necessarily follow that subjects were using information about the spatial relationship between the two colored circles. An alternative strategy for solving the discrimination would be to focus exclusively on one half of the pattern and to use the color of the circle as the cue for the trial outcome. To render this strategy ineffective, we included additional patterns involving a third color in the discrimination. The three patterns that signaled one outcome are shown in the left-hand column of the lower panel of Table 1, and their counterparts that signaled the opposite outcome are shown in the other column. It would not be possible to solve all three discriminations by focusing on one half of each pattern. Instead, a complete solution of the discrimination depends on subjects' appreciating the spatial relationship between the two colors in each pattern. If the hippocampus is necessary for detecting the spatial relationship between the colors, then the control group, but not the lesioned group, should solve this discrimination. Upon the completion of this stage of the experiment, both groups received a reversal of the discrimination just described.

In contrast to expectations, the hippocampal lesions did not prevent subjects from solving the structural discriminations. The

Table 1

Patterns Used in the Structural Discrimination Tasks

\begin{tabular}{cc}
\hline AV-BH & BV-AH \\
BH-AV & AH-BV \\
CV-AH & CH-AV \\
AH-CV & AV-CH \\
BV-CH & BH-CV \\
CH-BV & CV-BH \\
\hline A-B & B-A \\
B-C & C-B \\
C-A & A-C \\
\hline
\end{tabular}

Note. Upper panel: Details of the 12 patterns that were used for a structural discrimination that required information about the color, A, B, or $\mathrm{C}$, and orientation, horizontal $(\mathrm{H})$ or vertical $(\mathrm{V})$, of lines to be bound together. Lower panel: Details of the six patterns that were used for a spatial structural discrimination involving three colors, A, B, or C, which filled adjacent circles. Patterns described in the same column of each panel signaled the same outcome. only indication of a difference between the performance of the two groups during these discriminations was a hint that responding was more vigorous by the sham-operated than the hippocampal group. In order for us to be able to look more closely at this possible difference between the groups, they received trace conditioning in the hope that the consequent reduction in the rate of key pecking by both groups would prevent a possible difference between their response rates being masked by a performance ceiling. In addition, there is evidence that hippocampal lesions disrupt trace conditioning in both rats (Beylin et al., 2001) and rabbits (Kim, Clark, \& Thompson, 1995).

\section{Method}

\section{Subjects}

The subjects were 30 experimentally naive adult pigeons (Columba livia) housed in pairs. They were allowed free access to water and grit throughout the experiment, and they had free access to food until food deprivation was initiated. Surgery was conducted 7 days after arrival in the laboratory, and food deprivation was initiated 14 days after surgery. Birds were reduced to $80 \%$ of their free-feeding weights by being fed a restricted amount of food each day. On training days, food was made available immediately after each experimental session. Pigeons were maintained in a light-proof room in which the lights were on for $14.5 \mathrm{hr}$ each day. They were tested on successive days, 5 days a week, at the same time during periods when the lights were on in their holding room.

\section{Apparatus}

The experimental apparatus consisted of eight pigeon test chambers $(30 \times 33 \times 35 \mathrm{~cm})$. Each contained a three-key pigeon panel (Camden Instruments Ltd., Loughborough, England), the center key of which was replaced by a $10.5 \times 8.0-\mathrm{cm}$ (height $\times$ width) clear Perspex panel that was hinged at the top. Pecks on the panel were detected by a reed relay that was operated whenever a magnet attached to its lower edge was displaced by a distance greater than $1 \mathrm{~mm}$. The midpoint of the panel was $24 \mathrm{~cm}$ above the floor of the chamber. A color TV with a $10.5 \times 8.0$-cm screen was located $2.0 \mathrm{~cm}$ behind the Perspex panel. Food was delivered by operating a grain feeder (Campden Instruments Ltd., Loughborough, England), which was located directly below the Perspex panel. The opening to the feeder measured $5 \mathrm{~cm} \times 6 \mathrm{~cm}$, and the midpoint of the opening was $9 \mathrm{~cm}$ above the chamber floor. The feeder was illuminated whenever grain was made available. The chambers were permanently lit during all experimental sessions by a $2.8-\mathrm{W}$ bulb, operated at $24 \mathrm{~V}$, located $2.5 \mathrm{~cm}$ above the top of the Perspex panel. Risc PC microcomputers (Acorn Computers Ltd. Cambridge, England), which were programmed in Arachnid (Paul Fray Ltd., Cambridge, England), were used for the control of events, the recording of responses, and the generation of stimuli on the TV screens.

\section{Surgery}

One group of 15 pigeons received bilateral aspiration lesions of the Hp-APH region. During the operation, subjects were anesthetized with isofluorane at concentrations appropriate for the species and held in a modified stereotaxic instrument. The skin was incised, and the bone overlying the hippocampus was removed. The relevant brain tissue was then aspirated under visual guidance, with reference to the Karten and Hodos (1967) atlas. The area of bone overlying the lesion was replaced with bone wax, and the area was cleaned and the skin sutured. The remaining pigeons were treated in the same way, except that the brain tissue was not removed.

\section{Procedure}

All subjects first received three sessions in which they were trained to eat from the food hopper whenever it was raised. The entire TV screen was 
dark for this training. All pigeons then received 12 sessions of autoshaping with a single stimulus that consisted of an orange circle with a diameter of $2.5 \mathrm{~cm}$ that was presented in the middle of the TV screen. The remainder of the screen was dark, and the entire screen was dark throughout every intertrial interval (ITI). Food was presented by operating the grain hopper for $4 \mathrm{~s}$ immediately after the orange circle was removed from the screen. During the first 6 sessions, the orange circle was presented on each trial for $5 \mathrm{~s}$, the ITI was $30 \mathrm{~s}$ (range $=20-45 \mathrm{~s}$ ), and there were 30 trials in each session. For the next 6 sessions, each presentation of the orange circle lasted $10 \mathrm{~s}$, the ITI was $65 \mathrm{~s}$ (range $=45-85 \mathrm{~s}$ ), and there were 48 trials in a session.

The two groups then received 12 sessions of training with two simple discriminations. Each trial consisted of a square with sides of $2.0 \mathrm{~cm}$ being shown in the middle of the screen for $10 \mathrm{~s}$. The square was filled entirely with a color-either red or green, or with 2.9-mm wide black and white stripes-either horizontal or vertical. Each of the four stimuli was shown 12 times in a session in a random sequence with the constraints that the same stimulus was not shown more than twice in succession and that no more than three reinforced or nonreinforced trials occurred in succession. For half the pigeons in each group, food was presented on trials when the square was red or it was filled with horizontal stripes, but not when it was green or filled with vertical stripes. The opposite relationships between the four patterns and food were used for the remaining birds. Procedural details that have been omitted were the same as for the final sessions of the autoshaping stage. Unless stated otherwise, the procedural details for the present stage remained in effect for the subsequent stages of the experiment.

The next stage, which lasted for 24 sessions, involved a biconditional discrimination based on the patterns shown in the middle panel of Figure 1. The subjects that had previously received, as signals for food, a red square or horizontal lines now received, as signals for food, a single square containing either alternating red and black horizontal lines or alternating green and black vertical lines. The square filled with either red vertical or green horizontal lines signaled the absence of food. The relationships between the four patterns and food were the opposite of those just described for the remaining birds in each group.

Patterns based on those shown in the right-hand panel of Figure 1 were used for a feature-binding, structural discrimination in the following 24 sessions. The 12 patterns listed in the upper panel of Table 1 were each presented four times in a session with the colors red, yellow, and blue serving as A, B, and C, respectively. The dimensions of the two squares that made up each pattern were the same as for the previous stages, and the midpoint of each pattern was coincident with the midpoint of the TV screen. The patterns were presented in a random sequence with the constraints that every pattern was shown once in each successive block of 12 trials and that no more than three reinforced or three nonreinforced trials could occur in succession.

Both groups then received a spatial structural discrimination. On each trial two circles measuring $1.5 \mathrm{~cm}$ in diameter were presented side by side in the middle of the TV screen separated by a gap of $0.5 \mathrm{~cm}$. The circles were never both the same color, and each of them could be red, yellow, or blue. Food was presented after the patterns listed in the left-hand but not the right-hand column in the lower panel of Table 1 for half of each group; the other half received food after the patterns listed in the right-hand but not the left-hand column. After 12 sessions, both groups received a reversal of the original discrimination for a further 18 sessions. Procedural details that have been omitted were the same as for the previous stage.

The final 12 sessions of the experiment involved conditioning with a circle that was $2.5 \mathrm{~cm}$ in diameter and that was cyan for 24 trials and magenta for 24 trials in each session. The ITI was $60 \mathrm{~s}$ (range $=40-80 \mathrm{~s}$ ). For half the pigeons in each group, presentations of the cyan circle lasted for $10 \mathrm{~s}$ and presentations of the magenta circle lasted for $5 \mathrm{~s}$. Food was presented on every trial, $10 \mathrm{~s}$ after the onset of the conditioned stimulus (CS), which meant there was a 5-s trace interval between the end of the magenta CS and the delivery of food. The trials were presented in a random sequence with no more than two trials of the same type occurring in succession. The remaining pigeons received the same treatment, except that the 5-s trace CS was cyan and the 10-s delay CS was magenta.

\section{Histology}

After completion of behavioral testing, the pigeons were deeply anesthetized using Euthatal $(200 \mathrm{mg} / \mathrm{kg}$ sodium pentobarbitol) and perfused transcardially with physiological saline and $10 \%$ (vol/vol) formol-saline. The brains were then removed and stored in formol-saline solution for at least $4 \mathrm{hr}$. The formol-saline solution was then replaced with $25 \%$ (wt/vol) sucrose solution, and the brains were allowed to become saturated in the solution for 24 to $48 \mathrm{hr}$ before being sectioned $(40 \mu \mathrm{m})$, mounted on slides, and stained with cresyl violet.

\section{Results}

\section{Histology}

The aspiration lesion removed the hippocampus and the area parahippocampalis in all operated pigeons. The left-hand column of Figure 2 shows the maximum and minimum extent of the lesions, and the central column shows the positions of the target brain regions in normal pigeons. The lesion extended throughout the rostrocaudal extent of the Hp-APH in all pigeons. There was sparing of the most rostral extent of the hippocampus in all pigeons. There was evidence of damage to the hyperpallium apicale and the mesopallium in the majority of lesioned pigeons, although the damage was minimal and did not extend to complete removal in any pigeon. All lesioned pigeons had damage to the dorsal aspect of the nidopallium and the dorsolateral corticoid region. Although there was some variability between pigeons in the extent of damage to these regions, this variability did not relate in a systematic fashion to the performance of the pigeons in any of the behavioral tests.

\section{Behavior}

There are several reports of the acquisition of autoshaping being impaired by damage to the hippocampus (Colombo et al., 2001; Reilly \& Good, 1989). Accordingly, the results from the initial stage of the present experiment were examined in some detail to look for a similar effect. The left-hand panel of Figure 3 shows the mean rate of responding by the two groups for the six sessions of autoshaping with a 5-s CS, and a further six sessions of autoshaping with the same CS but with its duration extended to $10 \mathrm{~s}$. The right-hand panel of the figure shows the results from the same stage of the experiment, but in terms of the proportion of trials in each session on which at least one response was recorded. Despite the use of these different measures of behavior, the pattern of results in each figure is remarkably similar. Acquisition occurred swiftly during the 5-s CS, and maintained a relatively stable asymptote for the remainder of this stage. There was no indication that autoshaping was more effective with the control group than the hippocampal group. Two separate two-way analyses of variance (ANOVAs) were conducted using either individual mean rates of responding or individual mean proportions of trials with a response, for each of the six sessions of the first stage. They revealed a significant effect of session, $F \mathrm{~s}(5,140)>34.7, p<$ .001 , but the effects of group and the interaction were not significant $(p s>.25)$. Similar analyses with the results from the six sessions of training with the 10 -s CS revealed the effect of session 


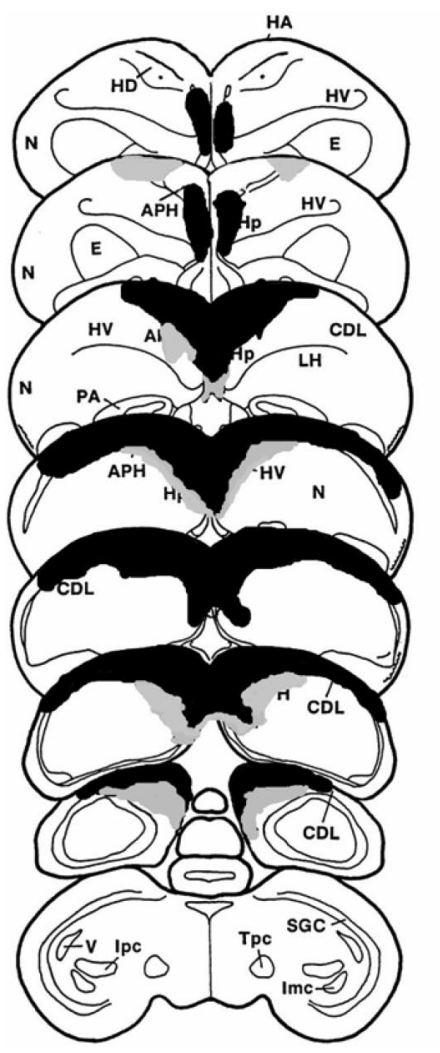

9.50

8.50

7.50

6.50

5.50

4.50

3.50

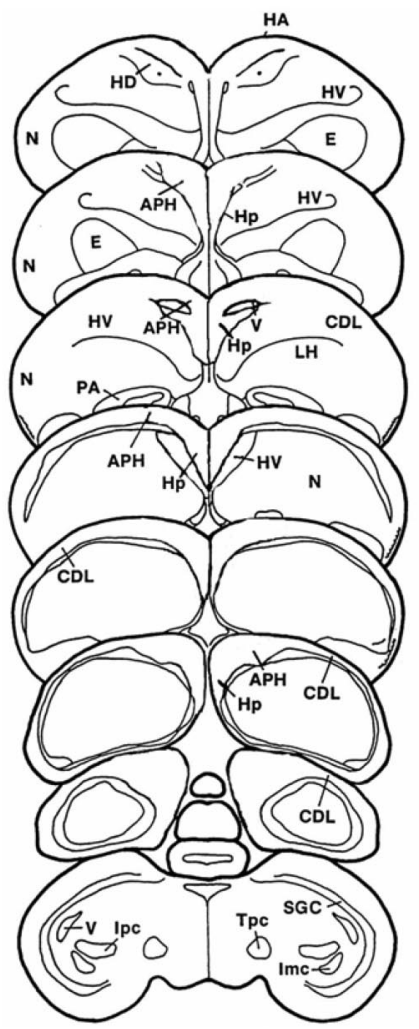

9.50

8.50

7.50

6.50

5.50

4.50

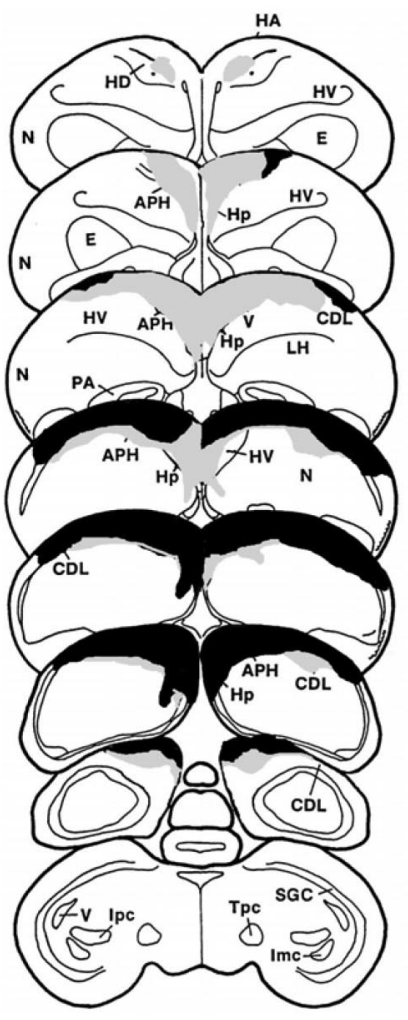

9.50

8.50

7.50

5.50

4.50

3.50

Figure 2. Reconstruction of the maximum (light gray) and minimum (black) extent of the lesions in hippocampus and area parahippocampalis (Hp-APH) lesioned pigeons from Experiment 1 (left-hand column) and Experiment 2 (right-hand column). The center column shows the position of the target brain regions in a normal pigeon. $\mathrm{HA}=$ hyperstriatum accessorium; $\mathrm{HD}=$ hyperstriatum dorsale; $\mathrm{HV}=$ Hyperstriatum ventrale; $\mathrm{N}=$ neostriatum; $\mathrm{E}=$ ectostriatum; $\mathrm{CDL}=$ area corticoidea dorsolateralis; $\mathrm{LH}=$ lamina hyperstriatica; $\mathrm{PA}=$ paleostriatum augmentatum; $\mathrm{SGC}=$ stratum griseum centrale; $\mathrm{V}=$ ventricle; Ipc $=$ nucleus isthmi, pars parvocellularis; $\mathrm{Tpc}=$ nucleus tegmenti pedunculo-pontine, pars compacts; Imc $=$ nucleus isthmi, pars magnocellularis. Reprinted from A Stereotaxic Atlas of the Brain of a Pigeon, H. J. Karten and W. Hodos, Figures 3.50, 4.50, 5.50, 6.50, 7.50, 8.50, and 9.50. Copyright 1967, with permission from Johns Hopkins University Press.
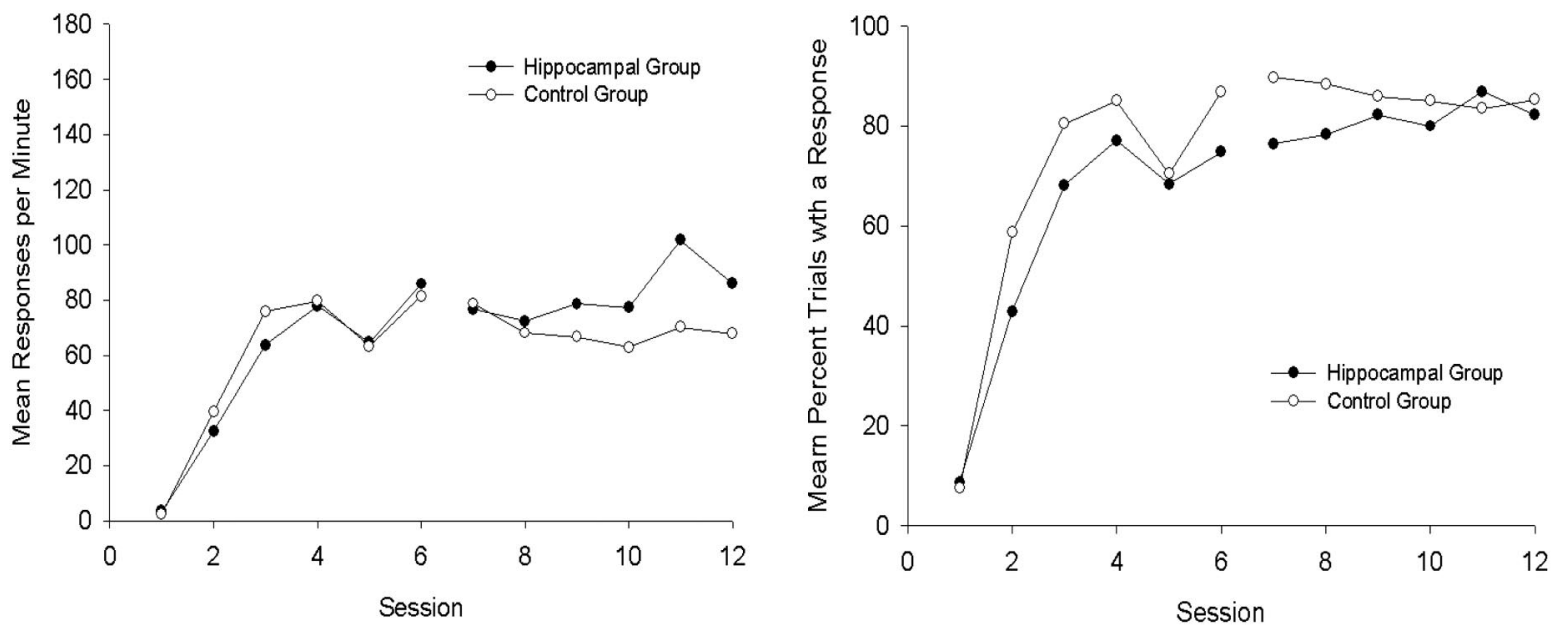

Figure 3. The mean rate of responding (left-hand panel) and the mean number of trials with a response (right-hand panel) for the hippocampal and control group during the autoshaping stage of Experiment 1. 
was significant when the individual mean rates of responding were analyzed, $F(5,140)=2.64, p<.05$, but not when the other measure of performance was analyzed $(p<.25)$. For both measures, the effects of group and the interactions were not significant $(p s<.25)$. In view of the failure to detect a significant influence of group, or Group $\times$ Session interaction, with either measure of behavior, all subsequent analyses focused on individual mean rates of responding in order to compare the performance of the two groups. Note that the conclusions drawn from these analyses are the same as those that would be drawn if they were based on individual proportions of trials with a response.

Simple discrimination. The effects of providing the groups with simple discriminations based on color and line orientation can be seen in the left- and right-hand panels, respectively, of Figure 4. Both groups acquired the discriminations in a similar fashion. Separate three-way ANOVAs were conducted for each discrimination. The Stimulus $\times$ Session interactions, $F \mathrm{~s}(11,308)>15.19$, $p s<.001$, were significant for both discriminations, and the effect of session was significant for the discrimination based on orientation, $F(11,308)=4.24, p<.001$, but not color $(p>.10)$. Simple main effects tests indicated that for both groups combined, responding was significantly faster during the reinforced than the nonreinforced trials in every session for the discrimination based on color, $F \mathrm{~s}(1,336)>6.71, p \mathrm{~s}<.01$, and all sessions except the first two for the discrimination based on orientation, $F \mathrm{~s}(1,336)>$ $4.46, p s<.05$. The remaining findings from the ANOVAs were not significant $(p s>.25)$

Biconditional discrimination. The results from the 24 sessions of training with the biconditional discrimination are shown, in 2-session blocks, in the left-hand panel of Figure 5. Both groups acquired this discrimination. There is a suggestion in the figure that responding was faster on both types of trial by the control than the hippocampal group, but statistical analysis indicated that this difference was not significant. A three-way ANOVA of individual mean rates of responding, in 2-session blocks, revealed that both the effect of session block and the Session Block $\times$ Stimulus interaction were significant, $F_{\mathrm{S}}(11,308)>4.90, p \mathrm{~s}<.001$. Tests of simple main effects indicated that responding by the two groups

Color Discrimination

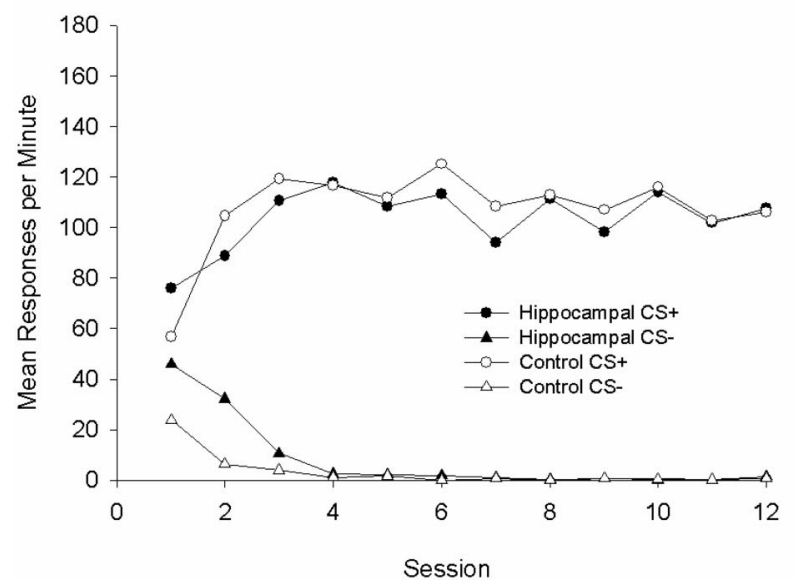

combined was significantly faster during the reinforced than the nonreinforced trials on each 2-session block, $F \mathrm{~s}(1,336)>6.05$, $p \mathrm{~s}<.05$. The remaining findings from the ANOVA were not significant $(p \mathrm{~s}>.10)$.

Feature-binding structural discrimination. The results from the first structural discrimination, which required feature binding for its solution, are shown in the right-hand panel of Figure 5. Both groups acquired the discrimination to a similar extent, and there is again a suggestion that responding was more vigorous by the control group than the hippocampal group. In keeping with the previous analyses, a three-way ANOVA of individual mean response rates in two-session blocks failed to reveal a significant effect of group or a significant interaction with this factor ( $p$ s $>$ $.10)$. The effect of session and the Session $\times$ Stimulus interaction were significant, $F_{\mathrm{s}}(11,308)>8.40, p \mathrm{~s}<.001$. Tests of simple main effects indicated that responding by the two groups combined was significantly faster on reinforced than nonreinforced trials on all but the 1 st four 2 -session blocks, $F \mathrm{~s}(1,336)>7.45, p \mathrm{~s}<.001$

Spatial structural discrimination. The left half of Figure 6 shows the acquisition, in 2-session blocks, of the spatial structural discrimination. Apart from a tendency by the control group to respond more rapidly than the hippocampal group, the performance of the two groups was quite similar. They both acquired the discrimination, and there was no indication that it was more demanding for one group than the other. The results from the reversal stage of the discrimination, again in 2-session blocks, are shown to the right in Figure 6. Again, there was a tendency for the control group to respond more rapidly than the hippocampal group, especially at the outset of the discrimination, but otherwise the performance of both groups throughout the reversal was quite similar. Thus, responding by both groups decreased to the patterns that previously signaled food and increased to those that had signaled its absence. A three-way ANOVA of individual mean response rates, in 2-session blocks, for the 12 sessions of the acquisition of the spatial structural discrimination revealed that the effects of session and the Stimulus $\times$ Session interaction were significant, $F \mathrm{~s}(5,140)=4.76, p \mathrm{~s}<.001$. Examination of the significant Stimulus $\times$ Session interaction with tests of simple 
Biconditional Discrimination

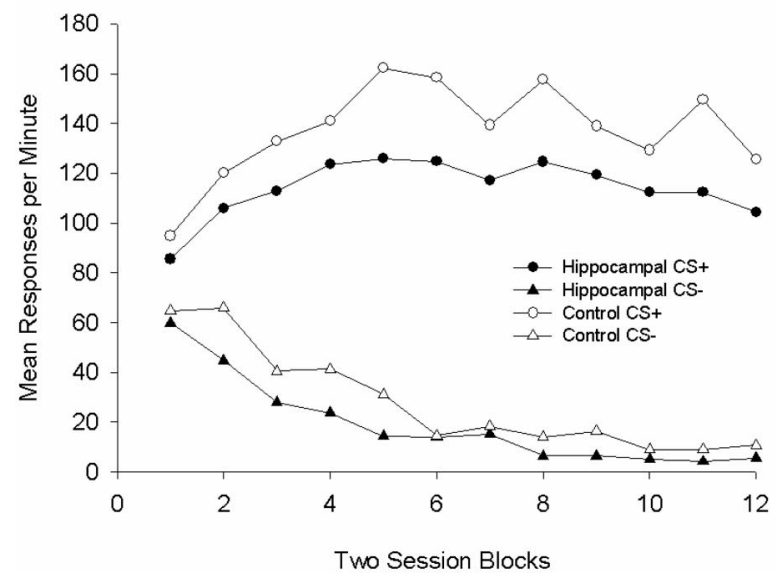

Feature-Binding Structural Discrimination

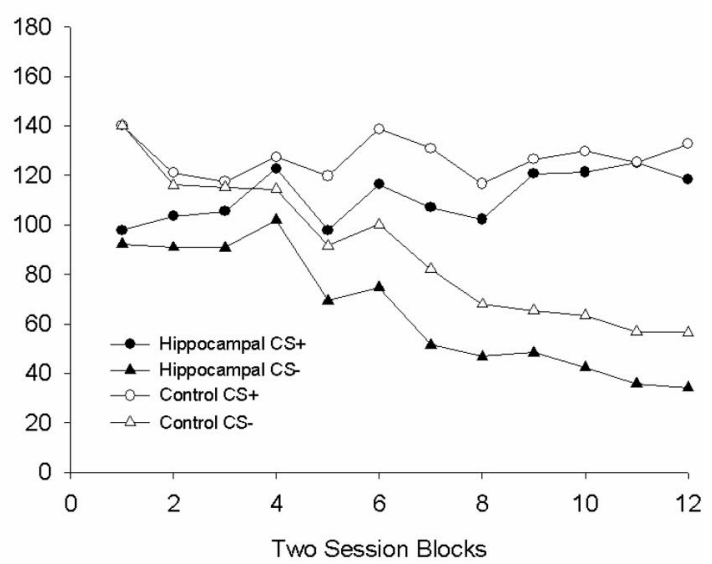

Figure 5. The mean rate of responding during reinforced (+) and nonreinforced (-) trials of the biconditional (left-hand panel) and the feature-binding structural (right-hand panel) discrimination by the hippocampal and control groups of Experiment 1 . CS $=$ conditioned stimulus.

main effects revealed that responding by both groups combined was significantly faster during the reinforced than nonreinforced trials from the second 2-session block onward, $F \mathrm{~s}(1,168)>4.00$, $p<.05$. The effect of group and all the interactions involving this factor were not significant $(p s>.10)$.

A similar three-way ANOVA for the results from the reversal stage revealed a significant Group $\times$ Session interaction, $F(8$, $224)=2.07, p<.05$. Subsequent tests of simple main effects revealed that this interaction was a consequence of response being significantly faster during both types of trial by the control than the hippocampal group during the second, two-session block of the reversal, $F(1,252)=3.95, p<.05$. The remaining findings from the three-way ANOVA were a significant effect of stimulus, $F(1$,
$28)=15.74, p<.001$, and of session, $F(8,224)=5.41, p<.001$, and a significant Stimulus $\times$ Session interaction, $F(8,224)=$ $20.88, p<.001$. Additional tests of simple main effects, to explore this last interaction, revealed that responding by both groups combined was significantly faster during the nonreinforced than the reinforced trials on the first 2-session block of the reversal, $F(1,252)=10.76, p<.01$, but significantly faster during the reinforced than nonreinforced trials from the fourth 2-session block onward, $F \mathrm{~s}(1,252)>5.23, p \mathrm{~s}<.05$. The effect of group and the remaining interactions involving this factor were not significant $(p s>.25)$.

Trace conditioning. Figure 7 shows for each group the mean rate of responding that was recorded during the 5-s trace CS, and

\section{Spatial Structural Discrimination}

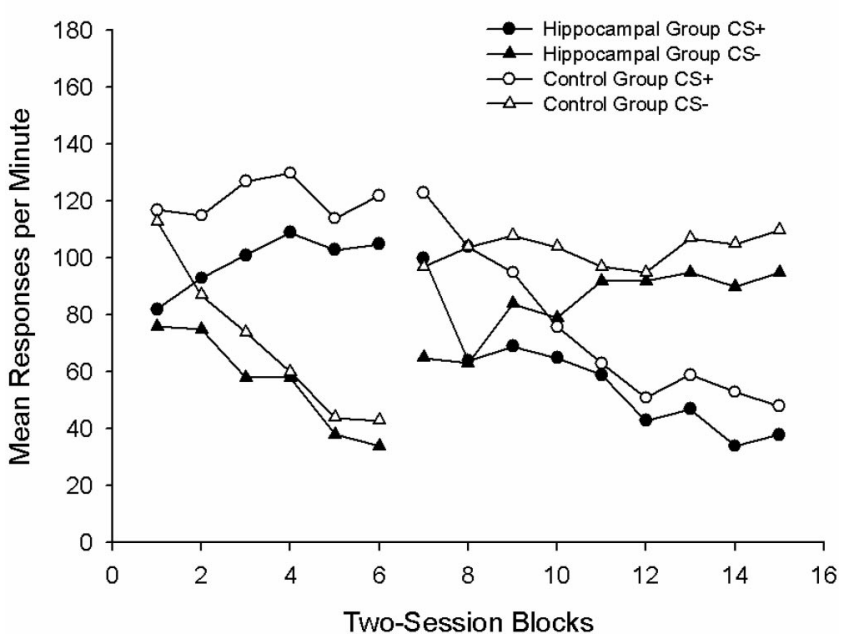

Figure 6. The mean rate of responding during reinforced (+) and nonreinforced (-) trials of the spatial structural discrimination by the hippocampal and control groups of Experiment 1. CS $=$ conditioned stimulus.

\section{Trace Conditioning}

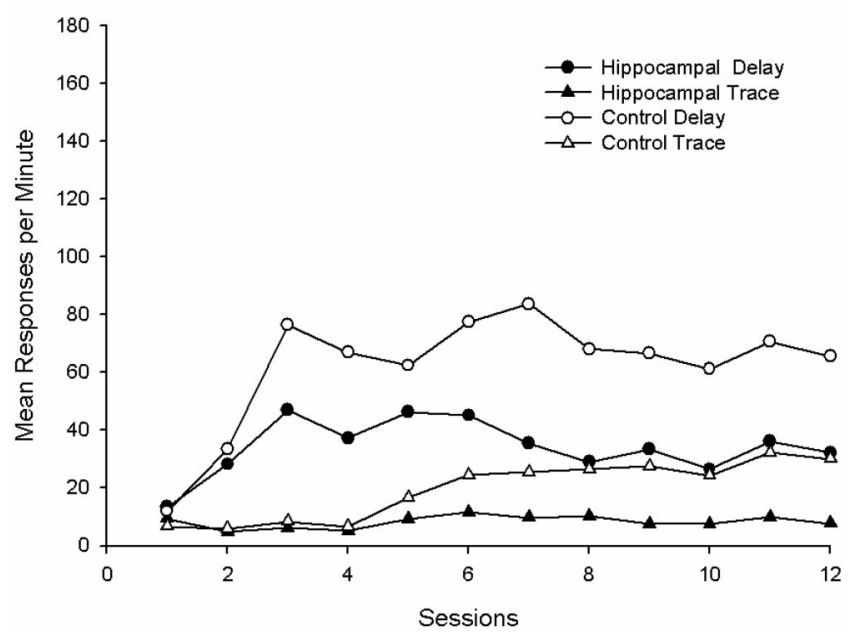

Figure 7. The mean rate of responding during delay and the trace conditioned stimulus by the hippocampal and control groups during the final stage of Experiment 1. 
the first $5 \mathrm{~s}$ of the delay CS for each of the 12 sessions of this stage. Each group responded more rapidly during the delay than the trace $\mathrm{CS}$, and responding during each of these stimuli was more vigorous by the control than the hippocampal group. A three-way ANOVA of individual mean response rates for each of the 12 sessions revealed a significant Group $\times$ Session interaction, $F(11$, $308)=2.63, p<.01$. Subsequent tests of simple main effects indicated that during both stimuli combined, responding was more vigorous by the control than the hippocampal group from Session 7 onward. To return to the ANOVA, we found that there were significant effects of session, $F(11,308)=6.60, p<.001$, and of stimulus, $F(1,28)=17.74, p<.001$, and a significant Session $\times$ Stimulus interaction, $F(11,308)=6.10, p s<.001$. The effect of group and the remaining interactions involving this stimulus were not significant $(p \mathrm{~s}>.05)$.

\section{Discussion}

The principal conclusion to be drawn from this experiment is that damage to the hippocampus did not impair the acquisition of a variety of visual discriminations, or the reversal of one of them. These findings join the results from other studies that have failed to find an effect of hippocampal lesions in pigeons on the acquisition of a visual discrimination (e.g., Broadbent \& Colombo, 2000; Good, 1987). The present results are, however, novel in showing for the first time that lesions of the hippocampus do not disrupt either a visual biconditional or a visual structural discrimination. Of course, these demonstrations involve null results, and it is conceivable that an alternative method of testing the groups would have revealed a difference between them. It is worth noting, however, that each group contained 15 pigeons. The failure to find an effect of the lesions on the acquisition of the discriminations is thus unlikely to be a consequence of the smallness of the groups.

The failure to detect an effect of hippocampal damage on the acquisition of the spatial structural discrimination is particularly striking in view of the clear evidence that this damage can influence the spatial behavior of pigeons. An early demonstration of this impairment was provided by Good (1987), who reported that although pigeons with hippocampal lesions are quite able to go to the correct arm of a T maze to find food, they do not perform so well on a reversal of this task (for a similar finding, see Broadbent $\&$ Colombo, 2000, who also found an impairment in the acquisition of this task). Other studies have shown that hippocampal damage impairs the capacity of pigeons to find food hidden in a large arena where its location is identified by reference to distant landmarks (Fremouw, Jackson-Smith, \& Kesner, 1997) or the shape of the arena (Vargas, Petruso, \& Bingman, 2004). Spatial behavior is also impaired when pigeons must find food by retrieving it from eight different locations in an open arena (Colombo Broadbent, Taylor, \& Frost, 2001; Colombo, Cawley, \& Broadbent, 1997). Thus, one implication of the present findings is that the hippocampus, at least in pigeons, is involved with processing some types of spatial information, but not others. Although this conclusion is potentially important, it depends on making comparisons of results from experiments conducted in different laboratories. In order to provide more compelling evidence that the hippocampus in pigeons processes only certain types of spatial information, the next experiment examined the effect of damage to this region on a variety of spatial tasks, including a structural discrimination.
Before turning to the next experiment, some comment is needed concerning our finding that autoshaping during the early stages of the experiment was unaffected by lesions to the hippocampus. This finding was unexpected in view of reports of hippocampal lesions resulting in a profound disruption of the acquisition of autoshaped key pecking by pigeons (Colombo et al., 2001; Reilly \& Good, 1989; Richmond \& Colombo, 2002). Colombo and Broadbent (2000) concluded that the reasons for the disruptive influence of hippocampal lesions on autoshaped key pecking are not known, which makes it difficult to interpret our failure to replicate this effect. One possible explanation for our results may relate to the nature of the response key. The response key for these earlier experiments was circular with a diameter of $2.5 \mathrm{~cm}$, whereas in the present study it was a rectangle that was $10.5 \mathrm{~cm}$ high and $8.0 \mathrm{~cm}$ wide. Richmond and Colombo (2002) found that pigeons with hippocampal lesions were more likely than controls to miss the response key when they pecked, and it is conceivable that the use of a larger response key enabled us to detect a greater proportion of the pecks that were actually made than is possible when a smaller key is used. Richmond and Colombo reported, however, that even when all the pecks made by the lesioned group are considered, they made fewer responses than the control group. This difference implies that some factor, in addition to the size of the response key, may have been responsible for the similar results from the two groups during initial autoshaping in the present study.

There was an indication from the biconditional discrimination onward that responding during reinforced and nonreinforced trials was slower in the hippocampal than the control group, but this difference was statistically significant only during the trace conditioning stage. Perhaps this deficit reflects the deficit reported in the earlier studies, but it required considerable training before it eventually became sufficiently large to enable a significant difference to be found between the groups during the final stage of the experiment. Of course, it is also possible that the significant difference between the rates of responding by the groups was a consequence of the introduction of trace conditioning. The trouble with this explanation is that it is not clear why the introduction of the trace conditioning trials should have resulted in slower responding during the delay CS by the hippocampal than the control group. If damage to the hippocampus impairs trace conditioning (Beylin et al., 2001; Kim et al., 1995), then there should have been no difference between the groups during the trials with the delay CS.

\section{Experiment 2}

The first phase of the second experiment was conducted in an arena so that the effects of damage to the hippocampus on tests of spatial memory could be assessed. For the first test, an array of eight pots containing food was placed on the floor of the arena, and subjects were required to retrieve food from every pot within a daily session. This task was based on a design developed by Spetch and Edwards (1986; see also Spetch \& Honig, 1988), which they regarded as a test of spatial working memory. Colombo et al. $(1997,2001)$ have shown that performance on this task is severely impaired in pigeons with damage to the hippocampus, and it was therefore anticipated that the present experiment would reveal a similar outcome. That is, a sham-operated control group was expected to make fewer errors of visiting pots from which food 
had already been retrieved than a group with damage to the hippocampus.

A second test in the arena was conducted with the eight pots in the same positions as for the previous test, but food was located in only one of them, and its position remained constant across trials. Prior to this test, training was given in which the single pot containing food was a distinctive color. The test trials were intended to be an assessment of spatial reference memory because the correct pot could be identified only by its spatial location, which remained unchanged from trial to trial. To our knowledge, this method of testing spatial reference memory in pigeons is novel. However, findings from a rather different study by Fremouw et al. (1997) suggest that the performance of the hippocampal group will be impaired relative to the control group. Pigeons were required to find food buried in a fixed location in a circular arena by reference to landmarks located at some distance from the food. It was found that the latency to find the food was substantially greater for subjects with hippocampal lesions than for control subjects.

The second phase of the experiment took place in conditioning chambers. The principal concern of this phase of the experiment was to test the reliability of the finding from Experiment 1 that hippocampal lesions do not impair the capacity to solve a spatial structural discrimination. If the performance of the hippocampal group should be disrupted on the spatial tasks conducted in the arena, but not on a spatial structural discrimination conducted in a conditioning chamber, then this would suggest that the hippocampus is involved in processing some types of spatial information, but not others. In an attempt to maximize the likelihood of finding an effect of the lesions, the discrimination was made more demanding than the one used for Experiment 1. In that study the gap between the two shapes (circles) containing the different colored squares was quite small, $0.5 \mathrm{~cm}$, but for the present experiment it was increased to $2.4 \mathrm{~cm}$. Instead of the two colors being presented in circles, they were presented in rectangles. This change was made because pilot studies have shown that normal pigeons can solve a spatial structural discrimination with rectangles of the same dimensions as those used in the present study, and a gap of $2.4 \mathrm{~cm}$ between them, but it is not easy. In fact, there was no indication of an impairment of the discrimination by the hippocampal group, and one further stage of training was administered to explore the generality and reliability of this finding by testing whether hippocampal lesions disrupt a spatial structural discrimination when food is delivered according to an instrumental rather than a Pavlovian schedule.

A secondary purpose of this phase of the experiment was to investigate further the finding from the previous experiment that the introduction of trace conditioning disrupted autoshaped key pecking to a greater extent in pigeons with Hp-AHP lesions than with sham lesions. Accordingly, upon completion of the initial autoshaping, both groups were trained in a similar manner to that used for the final sessions of Experiment 1. If performance during this stage should be inferior in the hippocampal than the control group, then it would indicate that it is not essential to give many sessions of training with various discriminations before the effects of hippocampal lesions on trace conditioning become evident. The results from the initial phase of training in this part of the experiment revealed rather weak responding by both groups during the trace CS. In an effort, therefore, to boost responding during this stimulus in the control group, and thereby provide more scope for revealing a deficit in the hippocampal group, the ITI was increased for a second phase of training. Balsam and Gibbon (1982) showed that this manipulation can enhance the rate of autoshaped key pecking during a trace CS. The spatial structural discrimination was introduced after the completion of the trace conditioning.

\section{Method}

\section{Subjects}

The 16 pigeons were from the same stock and housed in the same manner as for Experiment 1. Upon arrival in the laboratory, the birds were assigned at random, and in equal numbers, to the two groups. The surgery, the histology, and the treatment prior to behavioral testing was the same as for Experiment 1.

\section{Apparatus}

The working and reference memory experiments took place in a square room with walls that were $2.90 \mathrm{~m}$ in length and $2.59 \mathrm{~m}$ high. A plan of the room, together with the positions of the pots in which food could be placed, is shown in Figure 8. The black circles in the figure depict the positions of the eight food pots. They were arranged in the overall shape of a rectangle, with the distance between adjacent pots on the edge of the rectangle being $105 \mathrm{~cm}$. The two pots that were not on the edge of the rectangle were equidistant from the nearest four pots. The rectangular array was aligned at an angle of $22^{\circ}$ with the walls of the room. The distance between the top wall and the pot nearest to it, and between the right-hand wall and the pot nearest to it, was $25 \mathrm{~cm}$. The walls of the room were white, with colored posters on them, and the doors were green. A cloth screen, $1.8 \mathrm{~m} \mathrm{high}$, separated the test area from a region where the experimenter remained during every trial. The end of the screen near the door in the bottom left-hand corner of the room was hinged, which made it possible to close the screen behind the experimenter during a trial. A TV camera attached to the ceiling of the room was connected to a TV monitor in the region behind the screen so that the experimenter could observe the behavior of a pigeon throughout a trial. A desk light was placed on a shelf above the TV monitor with its $60-\mathrm{W}$ bulb positioned $30 \mathrm{~cm}$ above the screen partition. The food pots were $7.3 \mathrm{~cm}$ high and $7.5 \mathrm{~cm}$ in diameter. They were glued to blocks of wood that were $4.0 \mathrm{~cm}$ high, $9.0 \mathrm{~cm}$ wide, and $9.0 \mathrm{~cm}$ long. Each block was glued to the center of a white circular board with a diameter of 30.0

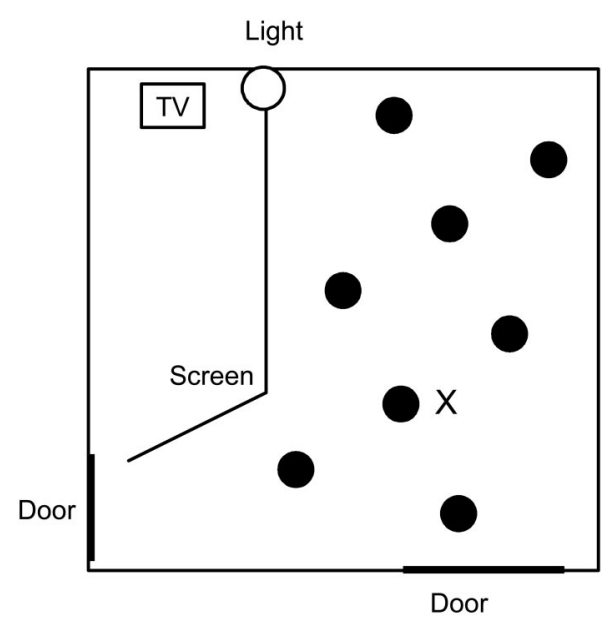

Figure 8. Plan of the experimental room used for the working and reference memory tasks in Experiment 2. The solid circles represent placement of the eight pots used in the experiment; $X$ (is adjacent to) the pot containing food. 
$\mathrm{cm}$ and a width of $1.0 \mathrm{~cm}$. The apparatus for the remaining stages of the experiment was that used in Experiment 1.

\section{Procedure}

To familiarize the birds with eating food from the pots, a single pot attached to its block of wood and circular board was placed in their home cages with a daily ration of food in it on each of 4 days. The pot remained in the cage until all the food had been eaten.

Working memory. All birds received three sessions of training designed to familiarize them with finding food in the arena used for the working memory task. On each of these days, the birds were released from the middle of the array of eight pots shown in Figure 8 and remained in the test arena until they had eaten all the food that had been placed in it. Two or three grains of dried sweet corn were placed on the white circular boards beneath four randomly selected pots for the first of these sessions. The same amount of food was then placed on the blocks beneath four pots for the second session, and food was placed in four pots for the third session. For the working memory task, a single grain of dried corn was placed in each pot and then a pigeon was placed in the center of the array with the light off. A low level of illumination was provided by the TV monitor. The desk light was turned on to start the trial once the experimenter was positioned behind the screen partition. The trial continued until the food in all eight pots had been eaten, or until $15 \mathrm{~min}$ had passed, at which point the desk light was turned off. Throughout the trial the experimenter kept a record of the number of visits a bird made to each of the eight pots. A visit was defined as any occasion on which both feet of the bird were placed on the white circular board beneath the pot. Unless a bird stood on the circular board in this manner, it was not possible for it to see whether the pot contained food. Training continued for eight sessions, with one trial in each session.

Reference memory. Pretraining for the reference memory task commenced on the day following completion of the working memory task. The two groups first received a single session in which they were required to eat their daily ration of food in their home cage from a pot that was identical to those used for testing, except that the pot, the block of wood, and the white circular board were painted blue. During the next four sessions of pretraining, the blue pot with four corn grains in it was placed by itself in the arena at the point marked $X$ in Figure 8. Pigeons were released from various points that were $1 \mathrm{~m}$ from the pot and remained in the arena until the corn had been eaten or until $10 \mathrm{~min}$ had passed. The final stage of pretraining lasted for four sessions and involved the array of eight pots shown in Figure 8. The blue pot, which was always positioned at the point marked $X$ on the figure, was different from the other seven pots, which were the same color, white, as for the working memory trials. There were four trials in each session, and for each trial a single grain of corn was placed in the blue pot. There were four release points, which were used once in a random sequence in every session. One release point was adjacent to the top wall in Figure 8 and equidistant from the two pots nearest to this wall. Two release points were adjacent to the right-hand wall and were equidistant from the two pots that were either nearest the top half or the bottom half of this wall. The fourth release point was adjacent to the bottom wall and equidistant from the two pots that were nearest to this wall. Birds were placed at the release point in the dark. Trials commenced when the experimenter turned on the light, and they ended when the grain of corn had been eaten or when $10 \mathrm{~min}$ had elapsed.

The treatment for the eight test sessions was identical to that just described, except that the blue pot was replaced by a white one that was identical to the other seven pots. Food was again located in the pot beside the point marked $X$ in Figure 8 .

Autoshaping. Eight days after the completion of the reference memory task, all birds received training in the conditioning chambers. All birds first received five sessions in which they were trained to eat from the food hopper whenever it was raised. The manner of this training was similar to that of Experiment 1. In each of 12 sessions, all pigeons then received autoshaping with a single stimulus that consisted of a white circle with a diameter of $2.5 \mathrm{~cm}$ presented at the middle of the TV screen. The remainder of the screen was dark and the entire screen was dark throughout every ITI. The white circle was presented for $10 \mathrm{~s}$, and food was made available for $4 \mathrm{~s}$ immediately after the circle was removed from the screen. The ITI was $60 \mathrm{~s}$ (range $=45-85 \mathrm{~s}$ ) and there were 48 trials in a session.

Trace conditioning. The next 23 sessions of the experiment involved conditioning in which a $2.5-\mathrm{cm}$ diameter circle was presented in the center of the TV screen for $5 \mathrm{~s}$ at a time. The circle was red for half the trials and green for the remaining trials. For half of the pigeons in each group, food was presented as soon as the red circle was removed from the screen and $5 \mathrm{~s}$ after the green circle was removed from the screen. The remaining pigeons received food immediately after the green circle and $5 \mathrm{~s}$ after the red circle. There were 20 trials with each of the red and green circles during the first 11 sessions of this stage, and 10 trials with each of these circles for the next 12 sessions. The ITI was $60 \mathrm{~s}$ (range $=45-85 \mathrm{~s}$ ) for the first 11 sessions and $180 \mathrm{~s}$ (range $=135-255 \mathrm{~s}$ ) for the remainder. The trials with the red and green circles were presented in a random sequence with the constraint that no more than three trials of the same type could occur in succession. One subject in the sham-operated control group ceased responding during this stage of the experiment; the data for this subject were therefore excluded from the analysis of the results.

Structural discrimination (Pavlovian conditioning). The next 18 sessions of the experiment involved discrimination training with patterns consisting of two rectangles that were each $24 \mathrm{~mm}$ high and $3 \mathrm{~mm}$ wide and separated by a gap of $24 \mathrm{~mm}$. The midpoint of the gap was coincident with the midpoint of the screen. The colors of the rectangles were red, green, and blue, and the manner in which these colors were assigned to the rectangles to create six different training patterns can be seen in the lower panel of Table 1. Details of the discrimination training were the same as for the spatial structural discrimination in Experiment 1, except that the ITI was $60 \mathrm{~s}$ (range $=35-85 \mathrm{~s})$.

Structural discrimination (instrumental conditioning). For the final stage of the experiment, the stimuli were based on those just described except that the gap between the rectangle was $0 \mathrm{~mm}$ and red was replaced with cyan, green with magenta, and blue with yellow. The patterns were presented for $15 \mathrm{~s}$. Responses that occurred during the first $10 \mathrm{~s}$ of any trial were counted but were otherwise without effect. On reinforced trials, the first peck that occurred $10 \mathrm{~s}$ after the onset of the stimuli resulted in the delivery of food. Pecks that occurred during the same period on nonreinforced trials were without a programmed effect. The remaining details of this training were the same as for the previous stage.

\section{Results}

\section{Histology}

The right-hand column of Figure 2 shows the maximum and minimum extent of the lesions for Experiment 2. The effects of the lesions were similar to those in Experiment 1.

\section{Behavior}

Working memory test. During the course of each trial, subjects could either approach pots that contained food, which were recorded as correct choices, or they could approach pots from which food had already been taken, which were defined as incorrect choices. In each session it was possible to make a maximum of eight correct choices. The number of incorrect choices made in a session was determined by the number of visits to empty pots, including revisits, that were made either before the subject had retrieved food from each of the eight pots or before the 15-min time limit for a trial had expired.

The left-hand panel of Figure 9 shows the mean number of correct and incorrect choices made by the two groups in each 

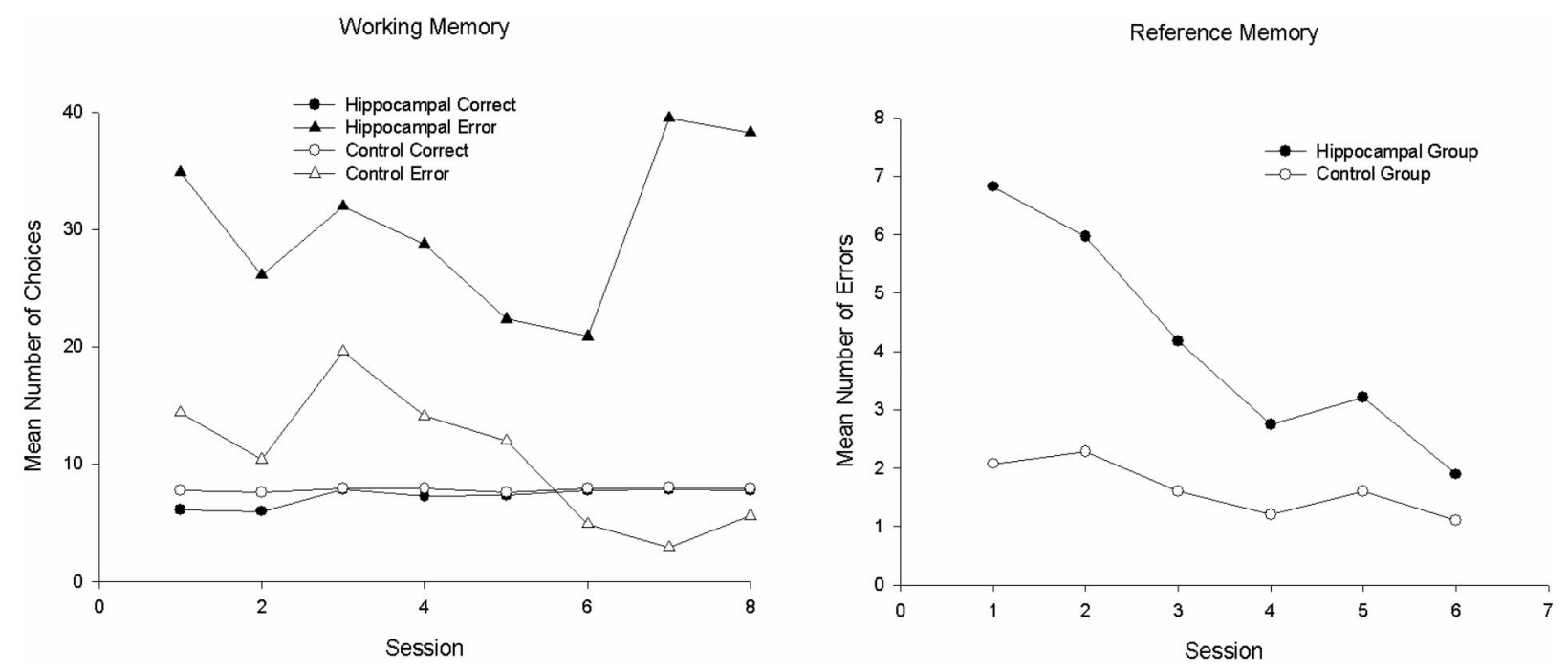

Figure 9. The mean number of correct and incorrect choices made during the working memory test (left-hand panel) and the mean number of errors made during the reference memory test (right-hand panel) by the hippocampal and control groups in Experiment 2.

session of the working memory test. Throughout this stage, both groups made a similar number of correct choices in each session, and by the end of training most subjects were making eight correct choices on every trial. The hippocampal group made consistently more incorrect choices than the control group, so that by the end of training the control group made fewer incorrect than correct choices whereas the opposite was true for the hippocampal group. Because so many subjects in each group made eight correct choices in each session, the results were analyzed using nonparametric tests. Comparisons of individual mean number of choices for the eight sessions combined revealed that the difference between the number of correct choices made by the two groups was not significant, Mann-Whitney $U(8,8)=18.0, p>.10$, but the difference between the number of incorrect choices was significant, $U(8,8)=1.0, p<.01$. In addition, analysis of individual mean number of choices made during the final two sessions revealed that the hippocampal group made significantly more incorrect than correct choices, Wilcoxon's $T(8)=0, p<.01$, whereas the sham-operated group made significantly fewer incorrect than correct responses, $T(8)=0, p<.01$.

Reference memory test. During the pretraining for this test, subjects were required to retrieve food from a blue pot that was always in the same position relative to an array of seven white pots. Both groups showed a strong preference for the blue over the white pots throughout this stage. The mean number of visits per trial to a white pot before choosing the blue pot was 1.32 for the hippocampal group and 0.86 for the sham-operated group. This difference between the groups was not statistically significant, $U(8,8)=17.0, p>.10$.

During the test stage of the spatial reference memory task, pigeons were required to find food in a single white pot that occupied the same location as the blue pot in the previous stage and that was surrounded by an array of seven identical white pots. The mean number of occasions on which the two groups chose a pot without food, including revisits, before choosing the correct pot is shown for every session in the right-hand panel of Figure 9.
The control group made fewer incorrect choices than the hippocampal group, but this difference between the groups diminished as training progressed. The difference between the groups was statistically significant, $U(8,8)=2.0, p<.01$.

Autoshaping. The mean rates of key pecking by the two groups during the 12 sessions of autoshaping with a single white circle are shown in the left-hand panel of Figure 10. In keeping with the previous experiment, the performance of both groups was similar. A two-way ANOVA of individual mean responses per minute with the factors of group and session revealed an effect of session $F(11,198)=10.03, p<.01$, but no effects of group and no Group $\times$ Session interaction $(p \mathrm{~s}>.25)$.

Trace conditioning. The mean rates of key pecking when conditioning was conducted with a delay CS and a trace CS are shown for each session for the two groups in the right-hand panel of Figure 10. The ITI was $60 \mathrm{~s}$ for the first 11 sessions and $180 \mathrm{~s}$ for the remaining 12 sessions. If hippocampal lesions disrupt trace but not delay conditioning, then the difference between the rates of responding during the delay and trace CS should be greater for the hippocampal than the control group. The figure shows this effect occurred in both stages of the experiment, but it was statistically significant only with the longer ITI. A three-way ANOVA of individual mean response rates during the initial 11 sessions of trace conditioning with the factors of group, session, and stimulus (delay or trace) revealed that the effect of group and the interactions involving this factor were not significant $(p s>.10)$. A similar analyses for the results from the final 12 sessions of this stage showed that the three-way interaction was significant, $F(11$, $154)=2.43, p<.01$. Simple effects analysis revealed significant Stimulus $\times$ Group interactions from Sessions 18 to $23, F_{\mathrm{S}}(1$, $165)>5.45, p s<.001$. Further analysis indicated that responding was significantly faster during the delay than the trace CS on each of these sessions for the hippocampal group, $F \mathrm{~s}(1,165)>21.56$, $p s<.001$, but not the sham control group, $(p>.05)$. The remaining findings from the ANOVA were a significant effect of session, $F(11,154)=2.40, p<.01$, and stimulus, $F(1,14)=$ 

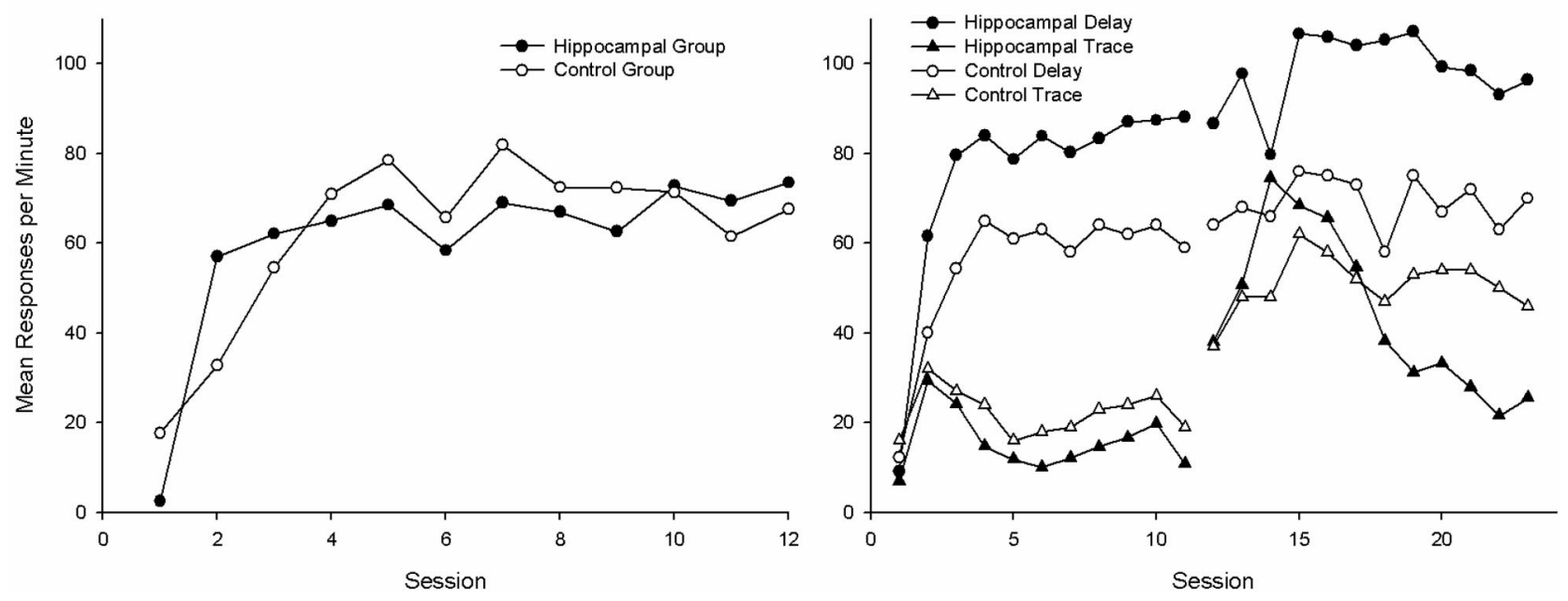

Figure 10. The mean rates of responding by the hippocampal and the control groups of Experiment 2 during the autoshaping stage (left-hand panel) and the trace conditioning stage with both a delay and a trace conditioned stimulus (right-hand panel).

22.13, $p<.001$. The Group $\times$ Stimulus, $F(1,14)=5.54, p<.05$, and the Stimulus $\times$ Session, $F(11,154)=2.37, p=.01$, interactions were significant. The effect of group and the Group $\times$ Session interaction was not significant $(p s>.25)$.

Structural discrimination (Pavlovian conditioning). The lefthand panel of Figure 11 shows the group mean rates of key pecking during the reinforced and nonreinforced trials for the 18 sessions of training with the first spatial structural discrimination. Although the discrimination appears to have been mastered more readily by the hippocampal than the control group, this conclusion was not supported by the outcome of a three-way ANOVA. The analysis revealed a significant Stimulus $\times$ Session interaction, $F(17,238)=3.24, p<.001$; all the main effects and remaining interactions were not significant $(p s<.10)$. Simple main effects analysis of the Stimulus $\times$ Session interaction revealed that responding by both groups combined was significantly faster on Sessions 11,13 , and 15 to $18, F \mathrm{~s}(1,252)>3.95, p \mathrm{~s}<.05$. Although not justified by the outcome of the analysis, tests of simple main effects were also conducted for the two groups separately. The purpose of this analysis was to confirm that the
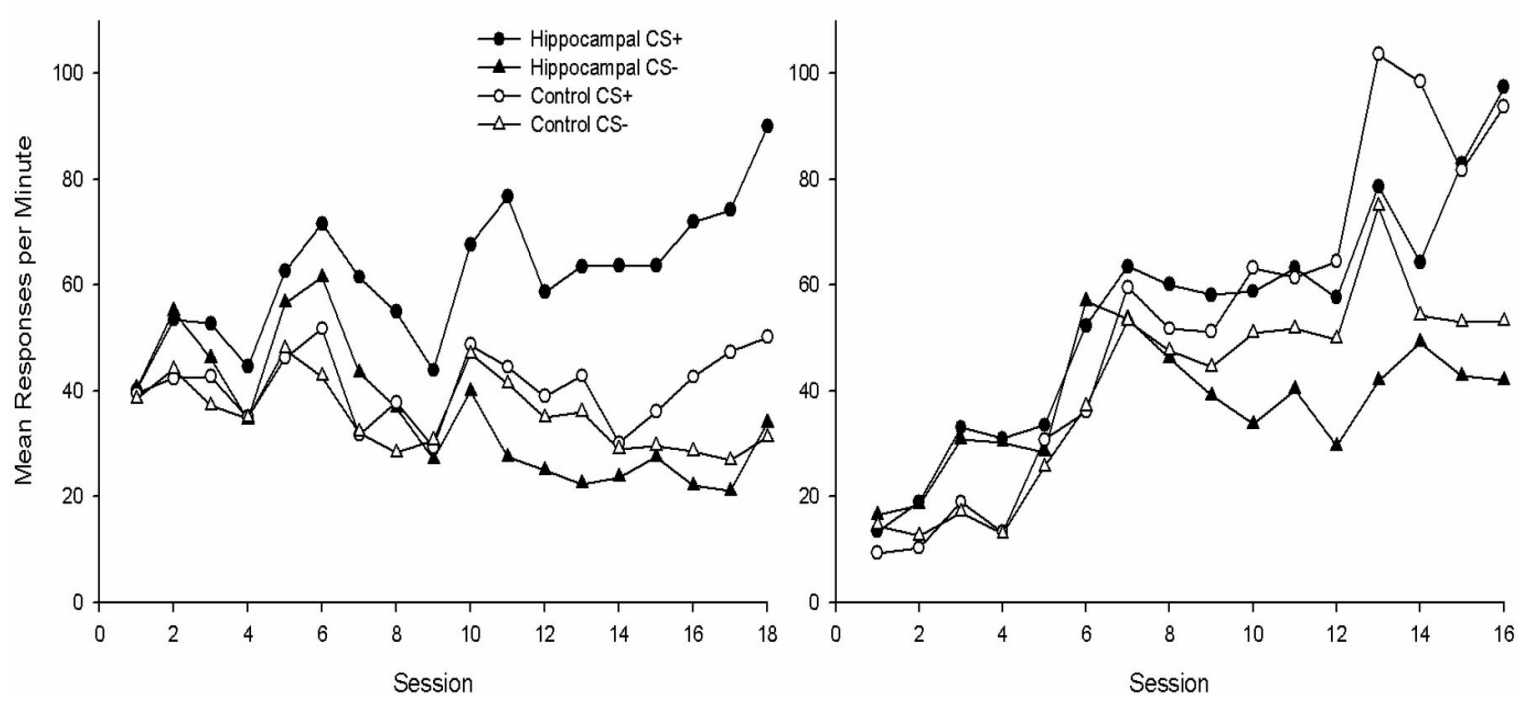

Figure 11. The mean rates of key pecking during the spatial structural discrimination of Experiment 2 during the autoshaping stage (left-hand panel) and the instrumental conditioning stage (right-hand panel). CS = conditioned stimulus, $+=$ reinforced, $-=$ nonreinforced. 
hippocampal group had indeed mastered the structural discrimination. There was a significant difference between the response rates during the reinforced and nonreinforced trials from Session 11 onward for the hippocampal group, $F_{\mathrm{s}}(1,252)>4.91, p \mathrm{~s}<.05$, whereas this difference was not significant on any session for the control group $(p s>.10)$.

Structural discrimination (instrumental conditioning). The right-hand panel of Figure 11 shows the mean number of responses per minute for the control group and hippocampal group during the first $10 \mathrm{~s}$ of the reinforced and nonreinforced patterns of the second spatial structural discrimination. Both groups mastered the discrimination. A three-way ANOVA of individual mean response rates revealed significant effects of session, $F(15,210)=8.13$, $p<.001$, and stimulus, $F(1,14)=8.89, p<.01$, and a significant Stimulus $\times$ Session interaction, $F(15,210)=7.42, p<.001$. The remaining interactions and the effect of group, however, were not significant $(p s>.25)$. Simple main effects analysis of the significant Stimulus $\times$ Session interaction revealed that responding was significantly faster on the reinforced than nonreinforced trials for both groups combined from Session 10 onward, $F_{\mathrm{S}}(1,224)>$ $4.99, p<.05$.

\section{Discussion}

In the initial phase of the experiment, pigeons received two spatial memory tasks. They were first required to retrieve food from each of eight pots in a fixed array for a working memory task, and then they were required to retrieve food from a single pot located in the same place among seven other pots for a reference memory task. Performance on both of these tasks was impaired by hippocampal lesions. It is important to note that during their training for the reference memory task, pigeons were required to find food in one blue pot among seven white pots. The hippocampal group made very few errors of approaching a white pot during this training, and its performance did not differ from that of the control group. The implication of this finding is that pigeons with hippocampal damage are quite capable of identifying a pot that contains food by relying on nonspatial information, such as its color, and ignoring other pots that are present in the test arena. The impairment that was seen in the hippocampal group on the two spatial tasks is thus unlikely to be a consequence of some theoretically uninteresting factor, such as an inability to refrain from approaching the nearest pot. Instead, the impairment can reasonably be said to have occurred because damage to the hippocampus affected the capacity to process spatial information.

The results from the second phase of the experiment indicate that the foregoing conclusion is in need of qualification. In keeping with the results from Experiment 1, there was no indication that damage to the hippocampus impaired performance on a spatial structural discrimination. This discrimination depends on subjects' being able to identify whether one object is to the left or right of another object, and it appears that an intact hippocampus is not essential for processing spatial information of this sort. In other words, the results from the two phases of the experiment indicate that the hippocampus in pigeons is essential for processing some sorts of spatial information, but not others. The implications of this conclusion are pursued in the General Discussion.

The left-hand panel of Figure 10 indicates that the magnitude of the discrimination between the two sets of patterns for the first structural discrimination was greater for the hippocampal than the control group. Indeed, there were several sessions when the hippocampal group responded significantly faster during the reinforced than nonreinforced patterns, and this was never true for the control group. The absence of any interaction with the main effect of group in the statistical analysis means, however, that it would be unjustifiable to conclude that the structural discrimination was mastered to a significantly greater extent by the hippocampal than the control group. Until there is statistically significant evidence to support this conclusion, it would perhaps be wise not to devote too much time to a discussion of why, at least numerically, hippocampal lesions appear to have improved the ability of pigeons to solve a spatial structural discrimination.

The results from the second part of the trace conditioning stage revealed that responding during the trace CS was substantially weaker than during the delay CS for the hippocampal but not the control group. In keeping with findings from rabbits (Kim et al., 1995; Solomon, van der Schaaf, Weisz, \& Thompson, 1986), and rats (Beylin et al., 2001; Shors, 2004), these results demonstrate that hippocampal lesions can disrupt trace conditioning. There was also an indication of this disruption during the first part of the trace conditioning stage, when the ITI was shorter than for the second part of this stage, but the effect was not statistically significant. Experiment 1 also revealed an effect of hippocampal lesions when subjects received training with a delay and a trace CS, but on this occasion the lesions resulted in a reduction in responding during both types of trial relative to that in the control group. It is hard to offer with any confidence an explanation for why responding during the delay CS was disrupted by hippocampal damage during the trace conditioning stage of Experiment 1, but not Experiment 2. However, the fact that the duration of the delay CS was $10 \mathrm{~s}$ in Experiment 1 and was $5 \mathrm{~s}$ in Experiment 2 may have been at least partly responsible for this difference between the experiments. Another puzzling aspect of the results from the trace conditioning stage is that numerically responding was faster during the delay CS by the hippocampal than the control group. Perhaps, through sampling error, the hippocampal group contained birds with a tendency to perform autoshaped key pecks during a delay CS more vigorously than those in the control group. This difference between the groups would then explain the high rate of responding by the hippocampal group during the first structural discrimination.

The discovery in both experiments that the trace conditioning stage revealed an effect of hippocampal damage is of significance for interpreting the results from the structural discriminations. It might be argued that damage to the hippocampus in pigeons affects their ability to deal with all aspects of spatial information and that the failure to detect this influence with a spatial structural discrimination was due to an insensitivity in the method of testing. The trace conditioning results pose a strong challenge this argument by showing that it was possible to detect an effect of hippocampal damage in the conditioning chambers. Instead of being due to an insensitivity in the method of testing, it is more likely that the failure to detect an effect of the hippocampal lesions on a spatial structural discrimination occurred because the hippocampus is not involved in processing the type of spatial information that is necessary for its solution.

In Experiment 1, considerable training was given before the structural discriminations were introduced. It is therefore possible that this extensive training was responsible for the failure to detect an influence of the hippocampal damage on the acquisition of these discriminations. The present results make such an explana- 
tion unlikely because subjects received relatively little training before they were given the spatial structural discrimination and yet there was no disruptive influence of the hippocampal lesions on its acquisition.

Apart from responding during the trace CS, there was no indication that the rate of autoshaped key pecking was reduced by damage to the hippocampus. This finding is consistent with the results from the previous experiment and is inconsistent with findings reported by Colombo et al. (2001); Reilly and Good (1989); and Richmond and Colombo (2002). We have already discussed possible reasons for our failure to replicate these earlier findings. All that has to be added here is that our failure appears to be reliable and that there is a need to understand why hippocampal lesions affect autoshaped responding in some circumstances, but not others.

\section{General Discussion}

The main purpose of the experiments was to explore the effect of damage to the hippocampus on the ability of pigeons to solve structural discriminations. Two such discriminations were used. In one, subjects were required to bind together information about the color and orientation of lines within a pattern in order to tell the difference between reinforced and nonreinforced patterns. In the other, subjects were required to discriminate between patterns on the basis of the spatial relationship between their components. There was no evidence of a disruptive effect of hippocampal lesions on either discrimination.

The failure to find an effect of hippocampal damage on the acquisition of a feature-binding structural discrimination stands in contrast to the claim that the hippocampus plays an important role in feature-binding in humans (Kroll et al., 1996; Mayes et al., 1999; Vargha-Khadem et al., 1997). Of course, the different tasks used with humans and pigeons, and the different types of damage to the hippocampus, make it virtually impossible to draw any conclusions from a comparison across these species. Nonetheless, the discrepancy between the conclusions drawn from the present studies and those drawn from studies with humans raises the possibility that the mammalian and avian hippocampus perform different roles in feature binding. The majority of research indicates that the avian and mammalian hippocampus have rather similar functions (e.g., Colombo \& Broadbent, 2000; MacPhail, 2002). It would thus be of considerable theoretical significance if it should transpire that, at least as far as feature binding is concerned, there is a difference in the function of the avian and mammalian hippocampus.

The absence of any effect of hippocampal damage on the acquisition of a spatial structural discrimination stands in marked contrast to the many findings with both mammals and birds that show the hippocampus is important for processing spatial information. The implication of these conflicting results is that the hippocampus is responsible for processing some sorts of spatial information but not others. If this conclusion is correct, it then becomes necessary to specify the type of spatial information that is processed by the hippocampus. One possibility is that this information must, in general, derive from large environments, such as the room in which our spatial memory tests were conducted, but not small environments, such as the conditioning chambers in which the spatial structural discrimination was administered. Alternatively, Haselgrove, George, and Pearce (in press) argued that the solution of a spatial structural discrimination depends on associations' being formed between the outcome of a trial and a template that functions as a "mental snapshot" of the pattern presented on the trial. Perhaps an intact hippocampus is not necessary for the acquisition of such a template. Furthermore, it is possible that when moving through an arena to find a cup in a particular location, pigeons rely on a different spatial representation to a template to identify the location of their goal. This representation could be based on the spatial relationships among different landmarks within the environment and the goal. Several authors have argued that the hippocampus is critical for learning about these relationships in both mammals (e.g., O'Keefe \& Nadel, 1978) and pigeons (e.g., Colombo, Cawley, \& Broadbent, 1997; Strasser \& Bingman, 1999).

If the avian homologue of the hippocampus is not involved in the discrimination of spatial structure, then what region might be? In addressing this question, it is interesting that Save et al. (1992) found that the ability of rats to detect that a familiar array of objects had been rearranged was disrupted by damage to either the parietal cortex or to the hippocampus. Perhaps, therefore, the avian homologue of the parietal cortex in rodents is involved in the processing of spatial structural information. There is evidence to suggest that the Wulst region in the pigeon may correspond to cortical regions in the mammalian brain (Medina \& Reiner, 2000), including the parietal cortex. Studies of the effects of lesions to subregions of the Wulst may thus offer an insight into the brain mechanisms underlying spatial structural learning in birds.

In conclusion, the experiments have revealed a number of novel findings. They have shown for the first time that hippocampal lesions do not undermine the capacity to solve structural or biconditional discriminations. It would thus appear that the avian hippocampus does not play a prominent role in the solution of either simple or complex visual discriminations, even when the discrimination is based on the spatial relationship between the components of a pattern. The experiments have also shown for the first time with pigeons that hippocampal lesions can disrupt trace conditioning. This finding extends the range of phenomena that are known to be affected by hippocampal damage in pigeons. In addition, it lends further support to the conclusion that the mammalian and avian hippocampus are functionally equivalent, because it has already been shown that damage to the hippocampus impairs trace conditioning in rats and rabbits. The novel theoretical conclusion that can be drawn from these experiments is that the avian hippocampus is involved in processing some types of spatial information, but not others. This region of the brain seems to be necessary for finding a hidden goal by reference to distant landmarks, but not for appreciating the spatial relationships between the components of a pattern. It remains to be seen whether this distinction is true for mammals.

\section{References}

Aggleton, J. P., \& Pearce, J. M. (2002). Neural systems underlying episodic memory: Insights from animal research. In A. Baddely, M. A. Conway, \& J. P. Aggleton (Eds.), Episodic memory: New directions in research (pp. 204-231). Oxford, England: Oxford University Press.

Balsam, P. D., \& Gibbon, J. (1982). Factors affecting trace decrements in autoshaping. Behaviour Analysis Letters, 2, 197-204.

Beylin, A. V., Gandhi, C. C., Wood, G. E., Talk, A. C., Matzel, L. D., \& Shors, T. J. (2001). The role of the hippocampus in trace conditioning: 
Temporal discontinuity or task difficulty? Neurobiology of Learning and Memory, 76, 447-461.

Bingman, V. P., Jones, T.-J., Strasser, R., Gagliardo, A., \& Ioalè, P. (1995). Homing pigeons, hippocampus and spatial cognition. In E. Alleva, A. Fasolo, H. P. Lipp, L. Nadel, \& L. Ricceri (Eds.), Behavioral brain research in naturalistic and semi-naturalistic settings (pp. 207-224). Boston: Kluwer Academic.

Broadbent, N., Gallagher, S., \& Colombo, M. (1999). Hippocampal lesions and negative patterning in pigeons. Psychobiology, 27, 51-56.

Broadbent, N. J., \& Colombo, M. (2000). Visual and spatial discrimination behavior following hippocampal lesions in pigeons. Psychobiology, 28, 463-475.

Colombo, M., \& Broadbent, N. (2000). Is the avian hippocampus a functional homologue of the mammalian hippocampus? Neuroscience \& Biobehavioral Reviews, 24, 465-484.

Colombo, M., Broadbent, N. J., Taylor, C. S. R., \& Frost, N. (2001). The role of the avian hippocampus in orientation in space and time. Brain Research, 919, 292-301.

Colombo, M., Cawley, S., \& Broadbent, N. (1997). The effects of hippocampal and area parahippocampalis lesions in pigeons: II. Concurrent discrimination and spatial memory. Quarterly Journal of Experimental Psychology: Journal of Comparative and Physiological Psychology, 50(B), 172-189.

Eichenbaum, H., \& Bunsey, M. (1995). On the binding of associations in memory: Clues from studies on the role of the hippocampal region in paired-associate learning. New Directions in Psychological Science, 4, $19-23$.

Fanselow, M. S. (2000). Contextual fear, gestalt memories and the hippocampus. Behavioural Brain Research, 110, 73-81.

Fremouw, T., Jackson-Smith, P., \& Kesner, R. P. (1997). Impaired place learning and unimpaired cue learning in hippocampal lesioned pigeons. Behavioral Neuroscience, 111, 963-975.

George, D. N., \& Pearce, J. M. (2003). Discrimination of structure: II. Feature binding. Journal of Experimental Psychology: Animal Behavior Processes, 29, 107-117.

George, D. N., Ward-Robinson, J., \& Pearce, J. M. (2001). Discrimination of structure: I. Implications for connectionist theories of discrimination learning. Journal of Experimental Psychology: Animal Behavior Processes, 27, 206-219.

Good, M. (1987). The effects of hippocampal-area parahippocampalis lesions on discrimination learning in the pigeon. Behavioural Brain Research, 26, 171-184.

Haselgrove, M., George, D. N., \& Pearce, J. M. (in press). The discrimination of structure: III. Representation of spatial relationships. Journal of Experimental Psychology: Animal Behavior Processes.

Karten, H. J., \& Hodos, W. (1967). A stereotaxic atlas of the brain of a pigeon. Baltimore: Johns Hopkins University Press.

Kim, J. J., Clark, R. E., \& Thompson, R. F. (1995). Hippocampectomy impairs the memory of recently, but not remotely, acquired trace conditioned responses. Behavioral Neuroscience, 109, 195-203.

Kroll, N. E. A., Knight, R. T., Metcalfe, J., Wolf, E. S., \& Tulving, E. (1996). Cohesion failure as a source of memory illusions. Journal of Memory and Language, 35, 179-196.

Lee, I., Hunsaker, M. R., \& Kesner, R. P. (2005). The role of hippocampal subregions in detecting spatial novelty. Behavioral Neuroscience, 119, $145-153$.

MacPhail, E. M. (2002). The role of the avian hippocampus in spatial memory. Psicologica, 23, 93-108.

Mayes, A. R., Van Eijk, R., Gooding, P. A., Isaac, C. L., \& Holdstock, J. S. (1999). What are the functional deficits produced by hippocampal and perirhinal lesions? Behavioral and Brain Sciences, 22, 460-461.

Medina, L., \& Reiner, A. (2000). Do birds possess homologues of mammalian primary visual, somatosensory and motor cortices? Trends in Neurosciences, 23, 1-12.

O'Keefe, J., \& Nadel, L. (1978). The hippocampus as a cognitive map. Oxford, England: Clarendon Press.

O'Reilly, R. O., \& Rudy, J. W. (2001). Conjunctive representations in learning and memory: Principles of cortical and hippocampal function. Psychological Review, 108, 311-345.

Reilly, S., \& Good, M. (1989). Hippocampal lesions and associative learning in the pigeon. Behavioral Neuroscience, 103, 731-742.

Richmond, J., \& Colombo, M. (2002). Hippocampal lesions, contextual retrieval, and autoshaping in pigeons. Brain Research, 928, 60-68.

Save, E., Poucet, B., Foreman, N., \& Buhot, N. (1992). Object exploration and reactions to spatial and nonspatial changes in hooded rats following damage to parietal cortex or hippocampal formation. Behavioral Neuroscience, 106, 447-456.

Shors, T. (2004). Memory traces of trace memories: Neurogenesis, synaptogenesis and awareness. Trends in Neurosciences, 27, 250-256.

Solomon, P. R., van der Schaaf, E. R., Weisz, D., \& Thompson, R. F. (1986). Hippocampus and trace conditioning of the rabbit's classically conditioned nictitating membrane response. Neuroscience, 100, 729_ 744 .

Spetch, M. L., \& Edwards, C. A. (1986). Spatial memory in pigeons (Columba livia) in an open-field feeding environment. Journal of Comparative Psychology, 100, 266-278.

Spetch, M. L., \& Honig, W. K. (1988). Characteristics of pigeons' spatial working memory in an open field. Animal Learning and Behavior, 16, $123-131$.

Strasser, R., \& Bingman, V. P. (1999). The effects of hippocampal lesions in homing pigeons on a one-trial food association task. Journal of Comparative Physiology A, 185, 583-590.

Sutherland, R. J., \& Rudy, J. W. (1989). Configural association theory: The role of the hippocampal formation in learning, memory, and amnesia. Psychobiology, 17, 129-144.

Vargas, J. P., Petruso, E. J., \& Bingman, V. P. (2004). Hippocampal formation is required for geometric navigation in pigeons. European Journal of Neuroscience, 20, 1937-1944.

Vargha-Khadem, F., Gadian, D. G., Watkins, K. E., Connelly, A., Van Paesschen, W., \& Mishkin, M. (1997, July 18). Differential effects of early hippocampal pathology on episodic and semantic memory. Science, 277, 376-380.

Wan, H., Aggleton, J. P., \& Brown, M. W. (1999). Different contributions of the hippocampus and the perirhinal cortex to recognition memory. Journal of Neuroscience, 19, 1142-1148.

Received, April 15, 2005

Revision received June 30, 2005

Accepted June 30, 2005 